\title{
Defining an epidemiological landscape by connecting host movement to pathogen transmission
}

\author{
Kezia Manlove $^{1}$, Mark Wilber ${ }^{2}$, Lauren White ${ }^{3}$, Guillaume Bastille-Rousseau ${ }^{4}$, Anni Yang ${ }^{5}$, \\ Marie Gilbertson ${ }^{6}$, Meggan Craft ${ }^{7}$, Paul Cross $^{8}$, George Wittemyer ${ }^{9}$, and Kim Pepin ${ }^{10}$ \\ ${ }^{1}$ Utah State University \\ ${ }^{2}$ The University of Tennessee Knoxville Institute of Agriculture \\ ${ }^{3}$ National Socio-Environmental Synthesis Center \\ ${ }^{4}$ Southern Illinois University Carbondale \\ ${ }^{5}$ USDA-APHIS National Wildlife Research Center \\ ${ }^{6}$ University of Minnesota Twin Cities \\ ${ }^{7}$ University of Minnesota System \\ ${ }^{8} \mathrm{USGS}$ \\ ${ }^{9}$ Colorado State University \\ ${ }^{10}$ United States Department of Agriculture Animal and Plant Health Inspection Service
}

October 18, 2021

\begin{abstract}
Environment drives the host movements that shape pathogen transmission through three mediating processes: host density, host mobility, and contact. These processes combine with pathogen life-history to give rise to an "epidemiological landscape" that determines spatial patterns of pathogen transmission. Yet despite its central role in transmission, strategies for predicting the epidemiological landscape from real-world data remain limited. Here, we develop the epidemiological landscape as an interface between movement ecology and spatial epidemiology. We propose a movement-pathogen pace-of-life heuristic for prioritizing the landscape's central processes by positing that spatial dynamics for fast pace-of-life pathogens are best-approximated by the spatial ecology of host contacts; spatial dynamics for slower pace-of-life pathogens are best approximated by host densities; and spatial dynamics for pathogens with environmental reservoirs reflect a convolution of those densities with the spatial configuration of environmental reservoir sites. We then identify mechanisms that underpin the epidemiological landscape and match each mechanism to emerging tools from movement ecology. Finally, we outline workflows for describing the epidemiological landscape and using it to predict subsequent patterns of pathogen transmission. Our framework links transmission to environmental context, providing a scaffold for mechanistically understanding how environmental context can generate and shift existing patterns in spatial epidemiology.
\end{abstract}

Defining an epidemiological landscape by connecting host movement to pathogen transmission Kezia Manlove, Department of Wildland Resources and Ecology Center, Utah State University, Logan, UT, 84322, USA. kezia.manlove@usu.edu

Mark Wilber, Department of Forestry, Wildlife, and Fisheries, University of Tennessee Institute of Agriculture, Knoxville, TN, USA

Lauren White, National Socio-Environmental Synthesis Center, University of Maryland, Annapolis, MD, USA 
Guillaume Bastille-Rousseau, Cooperative Wildlife Research Laboratory, Southern Illinois University, Carbondale, IL, 62901, USA. https://orcid.org/0000-0001-6799-639X

Anni Yang, Department of Fish, Wildlife, and Conservation Biology, Colorado State University, Fort Collins, Colorado, 80523, USA; National Wildlife Research Center, United States Department of Agriculture, Animal and Plant Health Inspection Service, Wildlife Services, National Wildlife Research Center, 4101 Laporte Avenue, Fort Collins, Colorado, 80521 USA; Department of Geography and Environmental Sustainability, University of Oklahoma, Norman, OK, 73019, USA

Marie L. J. Gilbertson, Department of Veterinary Population Medicine, University of Minnesota, St. Paul, MN, 55108, USA; Wisconsin Cooperative Wildlife Research Unit, Department of Forest and Wildlife Ecology, University of Wisconsin-Madison, Madison, WI, USA; ORCID 0000-0003-2142-0795

Meggan E. Craft, Department of Ecology, Evolution, and Behavior, University of Minnesota, St. Paul, $M N, 55108, U S A$

Paul C. Cross, U.S. Geological Survey, Northern Rocky Mountain Science Center, Bozeman, MT, 59718, $U S A$

George Wittemyer, Department of Fish, Wildlife, and Conservation Biology, Colorado State University, Fort Collins, Colorado, 80523, USA

Kim M. Pepin, National Wildlife Research Center, United States Department of Agriculture, Animal and Plant Health Inspection Service, Wildlife Services, National Wildlife Research Center, 4101 Laporte Avenue, Fort Collins, Colorado, 80521 USA

This draft manuscript is distributed solely for purposes of scientific peer review. Its content is deliberative and predecisional, so it must not be disclosed or released by reviewers. Because the manuscript has not yet been approved for publication by the U.S. Geological Survey (USGS), it does not represent any official USGS finding or policy.

Running title

Modeling the epidemiological landscape

Keywords

Transmission hotspot, epidemiological landscape, movement-pathogen pace-of-life hypothesis, spatial disease dynamics, mining-modeling approach, multipartite networks, movement mechanisms, host contact, host mobility

Article type : Perspective

Words in abstract : 199

Words in main text (excluding abstract, acknowledgements, references, table and figure legends)

$7101(+398$ in Box 1) = 7499

Words in each text box : Box $1=398$ words

Number of references : 184

Number of figures, tables, and text boxes: 6

Corresponding author

Kezia R. Manlove, 1595 N 1600 E Logan, UT, 84341, +1 (406) 600-2369, kezia.manlove@usu.edu

Statement of authorship 
All authors contributed to manuscript conceptualization. The framework was developed through a series of discussions among all coauthors. KRM built all figures and drafted the text. All authors contributed substantially across multiple rounds of manuscript revision.

Data accessibility statement

This paper contains no data.

\begin{abstract}
Environment drives the host movements that shape pathogen transmission through three mediating processes: host density, host mobility, and contact. These processes combine with pathogen life-history to give rise to an "epidemiological landscape" that determines spatial patterns of pathogen transmission. Yet despite its central role in transmission, strategies for predicting the epidemiological landscape from real-world data remain limited. Here, we develop the epidemiological landscape as an interface between movement ecology and spatial epidemiology. We propose a movement-pathogen pace-of-life heuristic for prioritizing the landscape's central processes by positing that spatial dynamics for fast pace-of-life pathogens are bestapproximated by the spatial ecology of host contacts; spatial dynamics for slower pace-of-life pathogens are best approximated by host densities; and spatial dynamics for pathogens with environmental reservoirs reflect a convolution of those densities with the spatial configuration of environmental reservoir sites. We then identify mechanisms that underpin the epidemiological landscape and match each mechanism to emerging tools from movement ecology. Finally, we outline workflows for describing the epidemiological landscape and using it to predict subsequent patterns of pathogen transmission. Our framework links transmission to environmental context, providing a scaffold for mechanistically understanding how the environment can generate and shift existing patterns in spatial epidemiology.
\end{abstract}

\title{
Introduction
}

Environment shapes the spatial, temporal, and behavioral structure of host movements and pathogen persistence, leading transmission to occur in some locations but not in others. The particular role the environment plays depends on the movement ecology of the host and pathogen: wildlife hosts can shed pathogens as they track resources across the landscape; livestock pathogens can jump abruptly between habitat patches as hosts are transported from farm to feedlot; and transmission of human pathogens often shows spatial signatures associated with work, caregiving, and social engagement. Environment also structures disease management efforts targeting specific locations that could give rise to super-spreading events or transmission hotspots, curtail the spread of invading epidemic "waves", or capitalize on predictable fluctuations in local transmission patterns.

However, despite these strong implicit connections, our understanding of the environment-movementtransmission interface remains in its infancy. Although movement ecology offers an array of tools that could help integrate environment, host movement, and pathogen deposition, the tools' outputs are somewhat detached from critical aspects of spatial transmission and some important components of spatial transmission are largely overlooked. Moreover, it is unclear how to best integrate the various processes each tool infers for optimal epidemiological predictions. Quantifying the environment's role in spatial dynamics of transmission, appropriately combining constituent processes, and identifying relevant mechanisms will require a stronger integration of movement ecology into conventional epidemiological frameworks.

There is a reason why movement and disease ecology developed separately to this point: they are interested in fundamentally different relationships. Movement ecology usually explores how individuals interact with their environments (Nathan et al. 2008), whereas disease ecology focuses on how individuals interact with one another to allow for pathogen transmission. Scaling individual-environment interactions up to predict individual-individual interactions is a long-standing challenge, and one that potentially distracts from other transmission-related insights that movement ecology has to offer. That lacuna is symptomatic of a broader interdisciplinary divide: the spatial modeling community lacks a systematic way to incorporate mechanistic 
and empirical insights from movement ecology into spatial models of transmission while retaining tractability and transportability beyond the specific system or landscape at hand. This gap impedes our ability to identify general principles about how the environment - both the local habitat and its broader scale landscape structure - shapes spatial patterns of pathogen transmission in real-world environments.

Spatial transmission dynamics depend on three central processes: host density processes that describe where hosts are located across the landscape, mobility processes that describe when and how hosts move among sites, and contact processes that describe the frequency, duration and form of host-host interactions and how those interactions relate to rates of pathogen transmission. Together, the host density, mobility, and contact processes combine with pathogen life-history to form an epidemiological landscape that interacts with the local volume of infected hosts to determine epidemiological dynamics through space and time (Fig. 1). Because it does not incorporate the volume of infected individuals directly, the epidemiological landscape exists apart from any specific disease event. Instead, it summarizes the potential for particular spatial transmission patterns to arise in a given environment on the basis of local host density, mobility, and contact. Identifying how density, mobility, and contact relate to the environment, however, is an open challenge that movement ecology can help to address.

Here, we propose a strategy to strengthen the interface between environment and transmission by focusing explicitly on the mechanisms and traits surrounding the epidemiological landscape. First, we use the epidemiological landscape to formalize the mechanistic relations that underpin transportable models. We then propose an heuristic that we refer to as the movement-pathogen pace-of-life hypothesis that suggests how to prioritize the density, mobility, and contact processes according to important pathogen life history attributes. Next, we identify a set of critical mechanisms determining the epidemiological landscape's structure, and match both the epidemiological landscape's central processes and our proposed mechanisms to metrics and inferential tools from movement ecology in sections organized around two research priorities (Fig. 2). The first priority is to infer specific aspects of pathogen transmission using existing movement ecology tools; the second is to determine how social context contributes to patterns of density, mobility, and contact. Finally, we outline three workflows that blend mechanisms with movement and transmission data to generate epidemiological forecasts that are transportable across landscapes and populations (Fig. 3), and end by identifying existing challenges and open questions.

\section{Using the epidemiological landscape to formalize the mechanistic relationships that underpin transportable models}

Transportable models - those that accurately predict spatial transmission patterns in landscapes and contexts apart from where they were developed - are both critically necessary and alarmingly absent at the spatiotemporal resolution necessary for disease management, especially in animal systems. Transportable models are especially important for systems where 1) spillover could occur across a huge geographic range, making boundary controls infeasible; 2) management actions shift depending on the specifics of the environmental context at hand; or 3) research is concentrated around particular pseudo-model systems but results are expected to extend to a wider suite of host-pathogen combinations.

Generating transportable predictions of spatial transmission is complicated because spatial disease dynamics rely on traits at two levels: traits that emerge from fundamental host-environment and pathogen pace-oflife mechanisms to shape the epidemiological landscape, and traits that emerge from the epidemiological landscape to influence spatial dynamics of transmission (Fig. 1). Which movement and transmission traits matter most depends on the relationship between host movements and pathogen life history. Spatial transmission traits like the occurrence of super-spreading events (Lloyd-Smith et al. 2005) or the pathogen's rate of spatial spread (Hallatschek et al. 2014) vary across environments, but explicit connections to underlying environmental attributes are rarely identified in practice. Risk assessments built from species-averaged life history or social ecology traits that make predictions at the scale of species ranges (e.g., Han et al. 2020) provide valuable insights for pathogens with slower paces of life whose transmission patterns mirror host presence or densities. Spatially explicit management of pathogens with faster life histories, however, requires higher-resolution predictions about exactly where transmission occurs and how far animals move 
while infected (Manlove et al. 2019).

When important spatial transmission traits are known, the epidemiological landscape can be constructed from those attributes directly through individual-based models that also account for the pathogen's life history. Unfortunately, appropriate information for building these models rarely exists in practice. Even when data are available, their ability to predict patterns in novel environments is often constrained through limited spatial replication and uncertainty about what constitutes an appropriate contrast set of transmission events that could have occurred but did not. In the absence of direct data describing spatial transmission, the epidemiological landscape must be predicted from knowledge about fundamental host-environment and pathogen-environment interactions, how those interactions vary across environments, and how the resulting variation governs key features of spatial transmission (Fig. 1).

Most methods that generate spatially explicit forecasts from underlying environments and animal movements cover only one facet of the epidemiological landscape, or extend to only a subset of individuals or times. While individual-based models can account for multiple processes and all individuals present, their predictions are laden with the builder's decisions about which processes to include. Even after predicting the epidemiological landscape, modelers must decide how to appropriately capture pathogen life-histories that allow transmission to occur across temporal lags or over longer distances in space.

Regardless of what approach one takes to model the epidemiological landscape, accurate out-of-sample prediction always depends on a clear-eyed consideration of appropriate detail, acknowledgement of functional responses that could hinder predictions outside the measured context, and a strong understanding of the system's fundamental mechanistic drivers. Identifying which processes matter most, and clarifying what factors help determine transportability across environmental and social contexts are critical first steps.

Prioritizing processes with the movement-pathogen pace-of-life hypothesis

Specific processes within the epidemiological landscape can dominate spatial patterns of transmission in particular situations. Which process dominates should be predictable on the basis of pathogen life history, providing a way to prioritize environment-movement-transmission inquiries and management within particular systems. Pathogens sample space according to their hosts' movements during infection or through extra-host movements across environmental media. As a consequence, the locations that a pathogen encounters are driven by the spatial ecology of its hosts, and by attributes of its own life-history like infectious period, mode of transmission, and ability to survive outside the host. Directly transmitted pathogens with short infectious periods and limited environmental persistence will disproportionately encounter locations that hosts visit immediately after contact events. That dependence should become less severe for pathogens with longer infectious periods or indirect modes of transmission. As a consequence, whether spatial transmission patterns are best predicted by host density, mobility, or contact will depend on the pathogen's life history.

We summarize this expectation through an heuristic that we call themovement-pathogen pace-of-life hypothesis . Under this heuristic, we expect pathogen intensity and spatial transmission risk to align with host density patterns when the pathogen's infectious period is long or modes of transmission are indirect; to align with host movement or contact patterns when the pathogen's infectious period is short or modes of transmission are direct; and to align with a convolution of host densities and environmental reservoirs when the pathogen's environmental persistence varies across environments. It follows that environmental drivers of density will be good predictors of transmission in the first case; environmental drivers of contact will be best in the second case; and densities should be modeled in interaction with environmental reservoirs in the final case. Mechanistic inquiries within a given system can be built forward on the basis of that prioritization.

Mechanisms connecting hosts, pathogens, and environments

Building transportable models requires understanding how the host, pathogen, and environment interface with one another, and how these interfaces combine in the context of the pathogen's pace-of-life. Articulating particular mechanisms that shape these relationships aids in their direct examination through a conventional 
movement ecology lens, but also suggests a set of measured features - entities like number of individuals within some neighborhood of a focal animal, or values describing environmental covariates surrounding a focal location - that could inform mining-modeling approaches (Han et al. 2020; Wijeyakulasuriya et al. 2020 ) or be built in as marginal constraints for multipartite networks to predict the epidemiological landscape (Manlove et al. 2018; Silk et al. 2018).

Some mechanisms are already well-understood. The propensity of hosts to form mass aggregations (LloydSmith et al. 2005; Cross et al. 2005) and stable social bonds (Sah et al. 2017) affects the contact process, as does the pathogen's mode of transmission and environmental persistence (Table 1). Synchrony of host life-history events can predictably alter host densities to produce seasonal pulses in transmission following an influx of new susceptible hosts (Peel et al. 2014). Density, mobility, and contact can also vary according to feedbacks between the pathogen and the host, either through physiological or behavioral shifts generated by the pathogen via direct or indirect routes (Hughes et al. 2011; Weinstein et al. 2018; Stockmaier et al. 2021; Table 1).

In order to expand beyond these mainstays, we compared treatment of host density, host mobility, and contact in spatial models of transmission for human and animal systems. Comparing human and animal approaches let us simultaneously capitalize on the largely top-down human and livestock research paradigms (which view host movements as arising primarily through the host's a priori knowledge), and the largely bottom-up animal movement research paradigm (which view host movements as arising primarily in response to local environmental context; Meekan et al. 2017; Miller et al. 2019).

In most spatial transmission models for humans and livestock pathogens, locations where individuals interact (e.g., houses, transit centers, feedlots) are assumed to be discrete and fixed through time (Keeling et al. 2001; Riley \& Ferguson 2006; Haw et al. 2020). Site-to-site mobilities depend on intervening distances and local and surrounding host densities (Viboud et al. 2006; Simini et al. 2012; Tizzoni et al. 2014). Once site-to-site movements occur, transmission-appropriate contacts are modeled according to local host density (following a functional form usually based on a priori knowledge about mode of transmission), and infection rates ultimately depend on the probability of transmission given appropriate contact.

These assumptions reflect aspects of human movement ecology that may not hold for free-ranging animals. Most humans are central place foragers (but see, e.g., Bharti et al. 2009), so assigning individuals to fixed locations and movement patterns has limited ramifications on model predictions. This permanent-pointresidence assumption is reasonable for some animal systems (e.g., nesting birds, denning wolves), but for others, residences are better represented as intensity surfaces, and even those representations may not be stationary through time. Host densities and site-to-site mobility rates are typically tied to the abundance, quality, timing, and spatial distribution of resources, along with the structure of the intervening landscape (Table 1). What constitutes a "resource", however, varies among hosts (for example, in humans, a critical resource is other humans; Miller et al. 2019), and knowledge of host ecology is critical for generating accurate predictions in animal systems. Humans also spend very little time in random walks (Table 1; Meekan et al. 2017). Instead, their movements are dominated by directed moves from a starting point to a pre-ordained destination. Environmental barriers like mountains and rivers rarely impede these movements (flight offers a similar ability to some animals; Table 1 ).

These human attributes occupy one extreme in host movement decision-making, while movement patterns derived from resource-driven random walks occupy the other. Most animal movement systems likely fall somewhere in-between. Perceiving these attributes as continuua and comparing the relative positions and movement dynamics of various species along them might inform a priori movement expectations that could streamline spatial transmission expectations and inform research, especially for understudied hosts.

Linking the environment to spatial ecology of individual hosts and pathogens

Host-environment and pathogen-environment interactions may produce reasonable predictions of spatial transmission for pathogens with slower paces of life and indirect modes of transmission. Applied analyses 
at the environment-movement-transmission interface have already proven useful in several of these systems (for example, in models of anthrax or brucellosis; e.g., Turner et al. 2014; Merkle et al. 2018 respectively), and a few extensions could expand their insights even further.

Biological underpinnings

The first step toward tailoring the epidemiological landscape to a particular environment is to identify what "resources" are most relevant to the host's movements and link those resources to patterns of host density and mobility (Hess 1996; White, Forester, \& Craft 2018). Resource distributions and accompanying habitat selection functions can provide the density predictions that we expect to dominate spatial transmission for pathogens with slower paces of life. However, these predictions may be insufficient for faster-paced pathogens with short infectious periods or behaviorally-specific transmission routes, whose transmission is more dependent on spatial patterns of contact and mobility. In these cases, a more targeted perspective may be necessary. There are at least four straight-forward pathways to incorporate additional complexity through mechanistic attributes of movement: account for variation in host internal state; account for environmentally-linked pathogen shedding; account for environmentally-linked persistence; and account for interactions between host movements and pathogen prevalence.

Movements arise from the interaction of the environment with the individual's internal state (Nathan et al. 2008). Individual internal states vary, however, and overlooking that variation (along dimensions including sex, age, reproductive status, and infection status) can lead models to miss systematic patterns of interindividual variation. Allowing movement estimates to vary according to basic individual attributes can help clarify signals and tailor movement predictions to the particular age, sex, reproductive status, or disease composition of the population at hand.

Transmission is sometimes tied to particular environmental conditions, through either host choice (e.g., if hosts preferentially engage in transmitting behaviors like scent marking, or mating in certain environments; Clontz et al. 2021), or direct environmental stimulation (e.g., if environmental irritants directly trigger coughs or other forms of excretion; Tarlo et al. 2016). When transmission is not distributed uniformly along the host's movement trajectory, spatial transmission patterns can be decoupled from aggregate patterns of host density or mobility. Residency times and return rates also inform expectations about system epidemiology (for example, the ratio of infectious period to return-to-residence times determines whether epidemics peak simultaneously across space; Keeling \& Rohani 2002).

Pathogen attributes also shape environmental persistence of some agents, leading contamination levels and acquisition rates to vary among environments even if local pathogen deposition rates are the same (Dorak et al. 2017). Forces like wind, water, or gravity can also allow for pathogen mobility outside the host in some cases (for example, cholera can diffuse along currents, Bertuzzo et al. 2010; and anthrax can diffuse down hills, Van Ness 1971). If environment-specific pathogen densities or physical connectivities are known, then they can inform local pathogen acquisition rates in forward simulation exercises predicting spatial patterns of transmission.

Host movement ecology sometimes interacts with pathogen prevalence to abruptly shift spatial patterns of transmission. Indirectly transmitted pathogens can accumulate in areas where hosts concentrate, leading to contamination levels that reduce long-term local densities (Boal \& Mannan 1999), or shift fitness landscapes enough to generate evolutionary consequences on host spatial phenotypes (Shaw et al. 2018).

Existing integrations with movement ecology

Individual-based models regularly incorporate density and mobility predictions from habitat- or step selection functions, respectively (e.g., Fortin et al. 2005; Avgar et al. 2016; Dougherty et al. 2018). Recent advances accounting for habitat in surrounding locations (Potts et al. 2014, Table 2) and variation in resource availability (Mysterud \& Ims 1998; Matthiopoulos et al. 2011; Moreau et al. 2011; Muff et al. 2020) are improving how well these models predict density and mobility in unsampled landscapes. Path segmentation methods that digest GPS movement trajectories into blocks of fixes with similar step lengths and 
turning angles (Table 2; Edelhoff et al. 2016) can differentiate among some forms of behavior, compare rates of engagement in those behaviors across host categories, and connect behavior changes to environmental and social contexts. Behavioral classifiers are improving with new data from cameras, animal-borne sensors, and other emerging technologies (Northrup et al. 2019; Table 2), offering new opportunities to link transmitting behaviors to specific environments. At the same time, methods describing residency times and return intervals (Pedersen et al. 2011) are able to connect time spent in random vs. directed search to particular environmental contexts.

\section{Next steps}

Integrating higher-resolution environmental drivers into spatial transmission predictions requires methods that explicitly account for spatial or temporal lags between pathogen deposition and acquisition (Fig. 1). While methods exist to model individual-individual associations through movement data, the resulting contact patterns are often specific to a user-specified definition of "contact". Methods that capture asynchronous contacts offer a framework to examine when and to what extent the definition of contact fundamentally alters expected spatial transmission dynamics.

A second task is to link the timescales of movement and movement data to the timescale of pathogen transmission. For example, dispersal and transmission kernels can describe the rates of spread of plant and animal invasions as functions of spatial covariates (e.g., Hefley et al 2017). However, kernels could be constructed from daily, seasonal, annual, or lifetime movements, and it is not obvious which timescale should be used for predicting spatial transmission.

Third, existing movement data are sometimes ill-suited to capture critical aspects of the epidemiological landscape, and constructing new or expanded datasets is sometimes imperative. Spatial transmission of pathogens with fast life histories will concentrate around locations of contact. If those locations are either missed because collared animals rarely encounter one another or biased due to the particular categories of hosts bearing collars, then spatial transmission forecasts may be underinformed or inaccurate. For pathogens that can move through environmental media outside the host, one could model environmental correlates of pathogen deposition, persistence and acquisition separately. For pathogens transmitted through specific behaviors, data on the spatial ecology of relevant modes of transmission, along with behavior-specific pathogen deposition rates, distances, and persistence times (Bourouiba 2021), may strengthen spatial predictions.

Fourth, movement predictions need to be able to update dynamically on the basis of host infection status. Distinct movement patterns among infected animals are easier to identify when we can reliably estimate timing of infection from individual pathogen loads or antibody titers. Antibody kinetic models (Borremans et al. 2016; Pepin et al. 2017; Prager et al. 2020), new biomonitoring technology that track temperature, acceleration, and febrile or respiratory responses (Hetem et al. 2008; Nathan et al. 2012; Adelman et al. 2014; Ahmed et al. 2016, Harari et al. 2017a; Harari et al. 2017b), and approaches from spatial phylodynamics (Biek et al. 2007; Kamath et al. 2016) all offer opportunities here.

Finally, spatial epidemiology may require some entirely new theory and methods. For instance, a richer framework for understanding the ecology of long-distance movements would be helpful, given the outsized role those movements play in the speed and structure of pathogen invasion (Hallatschek \& Fisher 2014; Wijeyakulasuriya et al. 2019).

Linking host social ecology to patterns of mobility and contact

Host contacts determine the spatial transmission patterns of directly transmitted pathogens, especially those with fast life-histories; and host contacts are driven by social, as well as spatial, factors. Social forces introduce a new layer of heterogeneity into the epidemiological landscape, especially if contacts are not randomly distributed across an animal's movements. Direct contact patterns for most species are likely much more overdispersed than one would expect if individuals moved independently and at random due to conspecific attraction. The influence of social attraction on the epidemiological landscape is not limited to contact, and social dynamics may also inform patterns of density and mobility. 
Epidemiological interest in social drivers of movement aligns well with a growing emphasis on social factors in movement ecology as a whole (van Beest et al. 2014). However, understanding where contacts occur and how each occurrence interfaces with the physical environment remains underexplored.

Biological underpinnings

At the population level, the environment can interact with host social ecology to fundamentally alter host density and mobility processes, often at annual scales. For example, environmentally-linked birth pulses can lead to seasonally-elevated densities, and compact breeding seasons can have important bearing on mobilities, especially among males seeking mates. Seasonal shifts in density and mobility can induce heterogeneity in persistence and spreading dynamics of pathogens with faster life-histories, but their effects will be more limited when pathogen life histories are slow. Other aspects of socially driven movement like dispersal may also shift with population-level host densities, though these shifts may also be tied to overall environmental resource availability.

Within populations, social factors often push local densities away from expectations based on individualenvironment interactions to generate spatial clustering. Spatially clustered hosts can produce temporal pulses in pathogen incidence, especially for pathogens with faster paces-of-life (Supplementary Text 1), and attributes like group size, stability, and social configuration are particularly important in shaping early epidemic progression in those systems (e.g., Cross et al. 2005; Sah et al. 2017; Bansal, Grenfell, \& Meyers 2007; respectively). Group size and environmental context are often interdependent. Different habitats may be optimal for groups of different sizes, leading to different habitat selection by larger groups than by smaller ones. If habitat selection varies with group size, then transmission events should concentrate at locations favored by larger groups, especially if transmission rates vary according to host density.

Group cohesion may also respond to environmental context, with important consequences on transmission in fast-paced pathogens. Hosts that live in stable social groups can exhibit contact processes that do not track with conventional density-dependent expectations, imposing a form of behaviorally mediated frequency dependence with knock-on effects for pathogen transmission (e.g., Cross et al. 2013; Manlove et al. 2014). Environmental correlates of group stability remain relatively underexplored (with the exceptions of environmentally driven soundscapes, lines-of-sight, and shared small space use, e.g., McComb et al. 2000; Strandburg-Peshkin et al. 2013; Pinter-Wolman et al. 2017 respectively). A better general understanding of how the environment relates to group cohesion and fission-fusion rates would be useful.

At an individual level, the social environment contains its own risks and resources which compete with environmental factors to shape host movement decisions. How socially driven movement affects transmission likely varies across systems, and potential mediating mechanisms could range from resource availability (Castillo-Contreras et al. 2018; Podgórski et al. 2013), to resource dispersion (Brandell et al. 2021), or seasonal shifts in social aggregation and sexual segregation (Wasserberg et al. 2009; Oraby et al. 2014). Human disease management activities can also disrupt the social environment, sometimes generating unintended consequences (Sokolow et al. 2019) through actions like culling (Donnelly et al. 2003) or translocation (Aiello et al. 2014).

Healthy animals may respond behaviorally to infected hosts (Croft et al. 2011) if they are cognizant of and responsive to disease presence, and some pathogens actively manipulate host behaviors to aid in their transmission (Koella, Sørensen, \& Anderson 1998; Vyas et al. 2007; Rosatte et al. 2006). Analogs to "social distancing" exist in a variety of non-human animal systems, accompanied by nuances including responses that vary in proportion to one's own susceptibility and social distancing exceptions for sick relatives (Stockmaier et al. 2021; Townsend et al. 2020). Pathogens with severe mortality burdens can also alter local host densities, shifting the epidemiological landscape through alterations to any and all density-dependent aspects of movement and transmission.

Existing integrations with movement ecology

Two arms of ecology - movement and behavior - are tackling questions about how social dynamics interface 
with patterns of host density, mobility, and contact. Movement ecologists are extending their individualenvironment framework to account for the presence of other individuals both in vivo (Jesmer et al. 2018) and in silico (Couzin et al. 2005; Couzin et al. 2011). Methods are emerging that allow spatial and social covariates to interact in common models of location and mobility (for example, in analyses of densitydependent habitat selection and its consequences, e.g., Webber \& Vander Wal 20181). At the same time, behavioral ecologists have built out their methods (which focus on individual-individual interactions and are thus often most applicable for models of contact) to account for spatial confounding. This is one motivation, for example, of pre-data permutation techniques, especially those that rely on group-incidence matrices (e.g., Farine 20135; Farine 2017). The behavioral emphasis, however, is often on causal inference about system organizing factors, not necessarily prediction to novel settings. Methods adjust for, but often do not explicitly draw inference to, environmental context.

As of now, scaling up from individuals to populations in both the movement and behavioral domains requires extrapolation across many upsampled animals. Spatial epidemiologists may benefit from workflows that focus more directly on group-level data that capture aspects of fission-fusion dynamics, and describe group compositional changes through time (e.g., Aureli et al. 2008; Palla et al. 2005; Figure 3).

\section{Next steps}

One urgent task for identifying social drivers of movement is to develop methods that can scale up from a subset of collared individuals to infer social and spatial dynamics across an entire population. If instruments are not deployed in proportion to local host densities, then areas with a higher ratio of collars to animals may be flagged as potential transmission hubs due to sampling alone. There are statistical methods for drawing inference from partially-observed contact data (e.g., Cross et al. 2013; Yang et al. 2021), but higher collar densities may also be necessary in some settings (Gilbertson, White, \& Craft 2021). Scaling up from individuals to populations often requires information about inter-individual attraction, along with auxiliary data on population densities or group sizes. Collaring studies are often designed to capture individual-environment, as opposed to individual-individual, interactions, but different design emphases may be necessary for questions about transmission (Plowright et al. 2019). Statistical guidance on how to best structure sampling designs to capture individual-environment vs. individual-individual interactions would be helpful.

A second task is to infer how social factors drive movement of instrumented animals. While it is feasible to include social covariates (both those describing the state of the focal individual and those describing the social environment) in standard state-switching or resource selection models, gathering appropriate social covariate data can be labor-intensive (Webber \& Vander Wal 2018; Albery et al. 2021). Co-occurring data on kinship, dominance, and affiliative histories that inform the stability of social bonds are rare, and concerted efforts to capture both locational and social covariates would strengthen our basis for understanding the social-spatial interface.

Bipartite networks can directly connect spatial and social interactions (Fortuna et al. 2009; Manlove et al. 2018), and a third task is to explore whether graph-based approaches could help infer spatial patterns of transmission. Bipartite structures form the basis of the spatially-embedded social networks that are widely used human epidemiological modeling (e.g., Balcan et al. 2009; Box 1). These models connect individuals to one another through shared space use. Individual association networks and spatial networks can both be built as projections of the bipartite structure to uncover locations with high levels of social connectivity.

Continuous-space mechanistic models (flocking dynamics models and their progeny) that allow spatial and social factors to interact directly also show potential (e.g., Dong et al. 2019; Buscarino et al. 2014; Levis et al. 2019). In real-world animal systems, researchers have used ideas from flocking dynamics to capture spatial and social mechanisms in individual-based models (IBMs; e.g., White, Forester, and Craft 2018; Pepin, Golnar, \& Podgórski 2021). Currently, these models are constrained by limited information on attributes like interindividual distances that are crucial for appropriate parameterization. Generating the requisite input parameters poses statistical challenges that are only beginning to be addressed (Richardson \& Gorochowski 2015; Hooten et al. 2018; Wilber et al. in review), forming a final important task for integrating social factors 
into spatial transmission modeling.

Modern workflows

Predicting the epidemiological landscape and emergent spatial transmission is a methodologically diverse objective, with approaches ranging from mechanistic tactics based on fundamental attributes of the system to more descriptive phenomenological approaches. New methods for Eulerian data (which capture aspects of space and time but are agnostic to individual identity) can infer transmission kernels as functions of the environment (Hefley et al. 2017), potentially expanding the utility of PDEs, semi-spatial, and metapopulation transmission models through methods like landscape homogenization (Box 1; Garlick et al. 2011). Eulerian approaches require data reflecting patterns that change over both space and time. This makes them especially useful for studying and predicting novel pathogen spread (especially for pathogens with slower paces-of-life or important environmental reservoirs), but less-useful for managing endemic transmission or designing mitigation strategies for pathogens whose spatial ecologies hinge on patterns of host contact. Here, we focus instead on workflows for Lagrangian data (which track location, time, and identity of focal animals, for example through GPS collars or other animal-borne sensors) that can inform the metapopulation and individual-based modeling approaches (Box 1) better-equipped to investigate the spatial dynamics of endemic pathogens and pathogens with faster paces-of-life.

Conventionally, researchers used Lagrangian data to identify drivers of spatial transmission by 1) correlating aspects of transmission with aspects of the environment; 2) building forward from those relationships to separately investigate key processes underlying spatial epidemiology; and finally 3) re-integrating processspecific inferences to generate predictions. Each of these steps has historically been ad hoc, and there is relatively little systematic guidance about which covariates to explore, which processes to prioritize, or how to properly integrate predictions across multiple processes. Errors can arise at each of these phases, but modern workflows offer new opportunities for reducing their impacts (Fig. 3).

All workflows begin with a preliminary correlative inquiry relating pathogen prevalence to environmental attributes (ideally, using existing datasets that track changes in hypothetically relevant environmental covariates). A brainstorming phase usually follows, during which researchers should consider the interplay of movement and pathogen pace-of-life, along with plausible mechanistic drivers and how they relate to the epidemiological landscape (Table 1). After this point, the workflows diverge.

In the mechanistic workflow, researchers then separately model density, mobility, and contact as functions of the physical environment, using distinct datasets and methods for each process (Table 2). Ideally, density should be characterized first and predicted over the spatial domain so that population fragmentation metrics derived from the density surface can be included as covariates in models of mobility. Contact, along with its relationship with both group size and engagement in transmitting behaviors, often requires distinct data derived from proximity loggers or direct observations. The epidemiological landscape can be constructed by predicting densities and subsequent mobilities on the basis of environmental covariates, and assigning site-specific transmission rates as functions of local environments and host densities. Predictions should be validated through pathogen data whenever possible, though mobility and density models can be validated using movement data alone where transmission data do not exist. The strength of the mechanistic workflow lies in its ability to draw causal inference between emergent movements and underlying drivers, which should improve resulting model transportability (but see Open Challenges: Extending outside the measured context below). Its fundamental weakness is that it is not sharply attuned to the social ecology of the host, so it may work best in systems where hosts move independently of one another and do not exhibit tight group formation or fidelity.

The multipartite network workflow places social and spatial connections on common footing from the start. This approach requires defining spatiotemporal cut-offs within which individuals are categorized as "associating" on the basis of a time lag defined by the pathogen's ability to persist in the environment. Individuals are then mapped to locations where associations occur, and then clustered to delineate spatial nodes. The individuals and spatial nodes are configured into a bipartite network (that potentially also tracks timing; 
Manlove et al. 2018), which can be projected down to generate unipartite individual and spatial networks. Uncertainty in network connection strengths can be reduced through application of existing (or cheaply available) spatial data on group sizes or habitat preferences that inform marginal degree distributions of both the spatial and the social graphs (Manlove et al. 2018; Cross et al. 2019). Location-specific transmission potentials could then be linked to environmental covariates and used to predict hotspots in novel settings. Whether forecasted hotspots actually harbor more host aggregations can be validated using local movement data, and whether those aggregations lead to transmission can be validated through pathogen data when those data exist. The strength of the multipartite network workflow is its balancing of spatial and social forces. Its fundamental weaknesses are its dependence on a collaring intensity high-enough to capture some contact events. Guidance regarding how to best discretize space and account for spatial autocorrelation is still emerging.

The final workflow builds from mining-modeling approaches for disease dynamics (Han et al. 2020). Data mining can be applied to a specific process within the epidemiological landscape, or to animal movement trajectories as a whole (Table 2; Fig. 3); we focus on the latter here. First, potential mechanistic drivers need to be translated into quantifiable "features" that can be measured along individual movement trajectories. These features (perhaps coupled with pathogen-associated features like prevalence) are used to train machine learning algorithms that are validated against a subset of withheld trajectories, and then used to forecast movements for all individuals. Density, mobility, and contact do not need to be treated separately here because each can be derived from the forecasted movement trajectories. Features can reflect both spatial and social processes (Table 2), and feature importance can help inform future research to isolate and test specific mechanisms. Movement forecasts can be adjusted for time lags derived from pathogen pace-of-life (e.g., Wilber et al. in review) to predict transmission. The strengths of the mining-modeling workflow are its ability to weight social and spatial factors in tandem, and its ability to scale up across individuals or landscapes. Its weaknesses lie in its dependence on appropriately identified features, its limited insights regarding causal mechanisms, and its abbreviated track record of application in movement ecology and spatial epidemiology.

Accurate prediction does not ensure effective intervention if the system's mechanistic drivers remain unknown. Mechanistically defining the epidemiological landscape can add insights that purely phenomenological multipartite network or mining-modeling approaches cannot. Ideally, the phenomenological workflows could be used in iteration with mechanistic approaches. For example, one could use ctmm and outputs from a method for capturing temporally lagged transmission to predict contacts, and then use mining approaches to link those inferred contacts to environmental context.

Open challenges

Connecting the epidemiological landscape's central processes

Pathogen transmission depends on many processes, and methods to integrate these processes and appropriately propagating error remain in short supply. This issue applies to both the mechanistic and the networkbased workflows, and can encumber some aspects of the mining-modeling approaches as well. Appropriately integrating processes should be a major focus for spatial epidemiological theory in the immediate future.

Identifying an appropriate level of detail

Which biological details to include depends on the resolution of available data and the effort's specific objectives. Mechanisms should be included if they are central to the overarching question or alter relevant predictions. Decisions about which biological details to include should precede decisions about model construction, since some approaches cannot capture certain mechanisms (Box 1). The relative timescales of host movements, environmental fluxes, and pathogen pace-of-life are also informative. Processes that change slowly relative to system epidemiology could be treated as constant; but processes that change quickly enough to affect disease incidence may need to be dynamic (Supplementary Text 1; Funk et al. 2015).

The temporal resolution of movement data is often under the researcher's control, though it trades off against 
device longevity through battery and memory capacity (Kays et al. 2015). Background knowledge about the timescales of relevant movements and behaviors can inform the timescale at which locations are recorded (Benhamou 2014; McClintock et al. 2014). Since rare, longer-distance moves are critical drivers of pathogen invasion speed, optimal disease invasion collaring might rely on slower fix rates and longer lifetimes as well as sampling across sex and age categories. On the other hand, extracting direct contacts from continuous time movement models will require much higher-resolution data, typically with fix rates in excess of one point per hour.

The spatial resolution of movement data is often determined by the particular technology involved, but researchers often control the spatial extent over which devices are deployed and thus the range of environmental covariates and density of collars within particular areas. Estimating environmental effects will be most efficient when the predictors vary substantially within the study's spatial domain, but if direct contacts need to be observed, then collar densities should remain high in some areas. Ideally, one should know the ratio of collars deployed to host density across the study. Habitat attributes that are consistent across the study area can be excluded from local predictions, but should be considered if the model is used to predict dynamics in novel contexts.

Finally, strengthening modeling infrastructure to allow for reliable inference often takes substantial effort (for example, through improved models of critical environmental covariates, increased performance of disease diagnostic tests, or refinements to statistical methodologies). Although this burden may diminish as methods improve, shortcutting variable development can be costly, and investment in variable development will nearly always be necessary to some degree.

Extending outside the measured context

While non-linear functional responses between environmental covariates and the epidemiological landscape's central processes do not preclude extrapolation, their presence should be considered when predicting in unsampled environments. Non-linearities are the rule, not the exception, in animal movement, and are expected from first principles alone (Avgar, Betini, \& Fryxell 2020). Meta-analyses of how habitat-selection, step-selection, or other attributes of movement vary across environments would help to generate baseline expectations about how availability affects density and movement, especially for hosts of common interest.

Disease feedbacks can also exhibit non-linear functional responses. A low-density population recovering from disease may exhibit fundamentally different rates of long-distance movement than nearby populations where densities are high; and age-specific mortality burdens can influence social structure, especially if mortalities concentrate among older and more knowledgeable individuals. The relationship between time since pathogen deposition and instantaneous rate of transmission may also be nonlinear (Almberg et al. 2011; Richardson \& Gorochowski 2015). This continuum remains underexplored (Breban 2013), leaving epidemiologists with no clear guidance about how to weight the force of infection arising from different modes of transmission over time.

Finally, predictions can fail in environments containing habitat types or social contexts that never arose in the training environment. Host populations with seasonal birth pulses may exhibit different habitat selection patterns than populations where birth pulses are diffuse, and habitats that are seasonally abandoned at some latitudes may be occupied year-round at others. In these cases, researchers may do well to take a more mechanistic approach to transportable modeling that draws from first principles of host biology.

ConclusionThe interface between movement and disease ecology offers exciting opportunities to improve spatial models of pathogen transmission and motivate work on understudied mechanisms shaping animal movement. Rapid advances and emerging workflows in both movement and disease ecology provide this interface a strong foundation and numerous opportunities for synergistic development to the advantage of both fields. However, shifts in emphasis are still needed to better define the epidemiological landscape and ultimately improve epidemiological forecasts and management. The epidemiological landscape, consisting of host density, mobility, and contact components, provides a conceptual bridge connecting spatial mechanisms to spatial dynamics of pathogen transmission and subsequent management. Focused inquiry around the 
upstream mechanisms and downstream phenomena surrounding the epidemiological landscape may reveal novel opportunities for targeted data collection, application of existing tools from movement ecology, and development of new methods and theory. We hope that this synthesis sparks additional discourse that can advance perspectives in spatial epidemiology and strengthen the bridge between movement and disease ecology.

\section{Acknowledgements}

Any use of trade, firm, or product names is for descriptive purposes only and does not imply endorsement by the U.S. Government.

\section{Literature Cited}

Adelman, J.S., Sahnzi C. Moyers, S.Z. \& Hawley, D.M. (2014). Using Remote Biomonitoring to Understand Heterogeneity in Immune-Responses and Disease-Dynamics in Small, Free-Living Animals. Integr Comp Biol,54 (3), 377-86.

Ahmed, S.T., Mun, H.S., Islam, M.M., Yoe, H. \& Yang, C.J. ( 2016). Monitoring activity for recognition of illness in experimentally infected weaned piglets using received signal strength indication ZigBee-based wireless acceleration sensor. Asian AustralJ Anim , 29 (1), 149.

Aiello, C.M., Nussear, K.E., Walde, A.D., Esque, T.C., Emblidge, P.G., Sah, P., Bansal, S. \& Hudson, P.J. (2014). Disease dynamics during wildlife translocations: disruptions to the host population and potential consequences for transmission in desert tortoise contact networks. Anim Conserv , 17, 27-39.

Albery, G.F., Kirkpatrick, L., Firth, J.A. \& Bansal, S. (2021). Unifying spatial and social network analysis in disease ecology. J Anim Ecol , 90 (1), 45-61.

Almberg, E.S., Cross, P.C., Johnson, C.J., Heisey, D.M. \& Richards, B.J. (2011). Modeling Routes of Chronic Wasting Disease Transmission: Environmental Prion Persistence Promotes Deer Population Decline and Extinction. PLoS One, 6 (5), e19896.

Altizer, S., Hobson, K.A., Davis, A.K., De Roode, J.C. \& Wassenaar, L.I. (2015). Do healthy monarchs migrate farther? Tracking natal origins of parasitized vs. uninfected monarch butterflies overwintering in Mexico. PLoS One, 10 (11), e0141371.

Aureli, F., Schaffner, C.M., Boesch, C., Bearder, S.K., Call, J., Chapman, C.A., Connor, R., Fiore, A.D., Dunbar, R.I., Henzi, S.P. \& Holekamp, K. (2008). Fission-fusion dynamics: new research frameworks. Curr Anthropol , 49 (4), 627-654.

Avgar, T., Potts, J.R., Lewis, M.A. \& Boyce, M.S. (2016). Integrated Step Selection Analysis: Bridging the Gap between Resource Selection and Animal Movement. Methods Ecol Evol, 7(5), 619-30.

Avgar, T., Betini, G.S. \& Fryxell, J.M. (2020). Habitat Selection Patterns Are Density Dependent under the Ideal Free Distribution.J Anim Ecol , 89 (12), 2777-87.

Balcan, D., Colizza, V. Goncalves, B., Hu, H., Ramasco, J.J. \& Vespignani, A. (2009). Multiscale Mobility Networks and the Spatial Spreading of Infectious Diseases. Proc Natl Acad Sci, USA 106 (51), 21484-89.

Bansal, S., Grenfell, B.T. \& Meyers, L.A. (2007). When Individual Behaviour Matters: Homogeneous and Network Models in Epidemiology.J R Soc Interface, 4 (16), 879-91.

Bastille-Rousseau, G., Douglas-Hamilton, I., Blake, S., Northrup, J.M. \& Wittemyer, G. (2018). Applying network theory to animal movements to identify properties of landscape space use. Ecol Appl, 28 (3), 854-864.

Becker, D. J., Streicker, D. G., \& Altizer, S. (2015). Linking anthropogenic resources to wildlife-pathogen dynamics: a review and meta-analysis. Ecol Lett, 18 (5), 483-495.

Benhamou, S. (2014). Of scales and stationarity in animal movements. Ecol Lett, 17 (3), 261-272. 
Berdoy, M., Webster, J. P., \& Macdonald, D. W. (2000). Fatal attraction in rats infected with Toxoplasma gondii. P R Soc Long D Bio, 267 (1452), 1591-1594.

Bertuzzo, E., Casagrandi, R., Gatto, M., Rodriguez-Iturbe, I. \& Rinaldo, A. (2010). On Spatially Explicit Models of Cholera Epidemics. J R Soc Interface, 7(43), 321-33.

Bhamidi, S., Van Der Hofstad, R., \& Komjathy, J. (2014). The front of the epidemic spread and first passage percolation. J Appl Probab, 51 (A), 101-121.

Bharti, N., Tatem, A.J., Ferrari, M.J., Grais, R.F., Djibo, A. \& Grenfell, B.T. (2011). Explaining seasonal fluctuations of measles in Niger using nighttime lights imagery. Science, 334(6061), 1424-1427.

Biek, R., Henderson, J.C., Waller, L.A., Rupprecht, C.E. \& Real, L.A. (2007). A High-Resolution Genetic Signature of Demographic and Spatial Expansion in Epizootic Rabies Virus. Proc Natl Acad Sci USA,104(19), 7993-1998.

Boal, C.W. \& Mannan, R.W. (1999). Comparative breeding ecology of Cooper's hawks in urban and exurban areas of southeastern Arizona. J Wildlife Manage, 77-84.

Borremans, B.,Hens, N., Beutels, P., Leirs, H. \& Reijniers, J. (2016). Estimating Time of Infection Using Prior Serological and Individual Information Can Greatly Improve Incidence Estimation of Human and Wildlife Infections. PLoS Comput Biol, 12(5), e1004882.

Borremans, B., Faust, C., Manlove, K.R., Sokolow, S.H. \& Lloyd-Smith, J.O. (2019). Cross-species pathogen spillover across ecosystem boundaries: mechanisms and theory. Philos T R Soc B , 374 (1782), p.20180344.

Bourouiba, L. (2021). The Fluid Dynamics of Disease Transmission. Annu Rev Fluid Mech 53 (1), 473-508.

Bracis, C., Bildstein, K.L. \& Mueller, T. (2018). Revisitation Analysis Uncovers Spatio-Temporal Patterns in Animal Movement Data.Ecography , 41(11), 1801-11.

Brandell, E.E., Dobson, A.P., Hudson, P.J., Cross, P.C. \& Smith, D.W. (2021). A Metapopulation Model of Social Group Dynamics and Disease Applied to Yellowstone Wolves. Proc Natl Acad Sci USA, 118(10).

Breban, R. (2013). Role of Environmental Persistence in Pathogen Transmission: A Mathematical Modeling Approach. J Math Biol , 66(3), 535-46.

Brewster, L.R., Dale, J.J., Guttridge, T.L., Gruber, S.H., Hansell, A.C., Elliott, M. et al. (2018). Development and application of a machine learning algorithm for classification of elasmobranch behaviour from accelerometry data. Mar Biol , 165 (4), 1-19.

Buscarino, A., Fortuna, L. Frasca, M. \& Rizzo, A. (2014). Local and Global Epidemic Outbreaks in Populations Moving in Inhomogeneous Environments. Phys Rev E, 90(4), 042813.

Calabrese, J.M., Fleming, C.H. \& Gurarie, E. (2016). Ctmm: An R Package for Analyzing Animal Relocation Data as a Continuous-time Stochastic Process. Methods Ecol Evol, 7(9), 1124-32.

Cassirer, E.F., Plowright, R.K., Manlove, K.R., Cross, P.C., Dobson, A.P., Potter, K.A. \& Hudson, P.J. (2013). Spatio-temporal dynamics of pneumonia in bighorn sheep. J Anim Ecol , 82(3), 518-528.

Castillo-Contreras, R., Carvalho, J., Serrano, E., Mentaberre, G., Fernandez-Aguilar, X., Colom, A., Gonzalez-Crespo, C., Lavin, S. \& Lopez-Olvera, J.R. (2018). Urban Wild Boars Prefer Fragmented Areas with Food Resources near Natural Corridors. Sci Total Environ, 615 (February), 282-88.

Charu, V., Zeger, S., Gog, J., Bjornstad, O.N., Kissler, S., Simonsen, L., et al. (2017). Human Mobility and the Spatial Transmission of Influenza in the United States. PLoS Comput Biol, 13(2), e1005382.

Clark, J. S. (1998). Why trees migrate so fast: confronting theory with dispersal biology and the paleorecord. Am Nat, 152(2), 204-224. 
Clontz, L.M., Pepin, K.M.,VerCauteren, K.C. \& Beasley, J.C. (2021). Behavioral State Resource Selection in Invasive Wild Pigs in the Southeastern United States. Sci Rep-UK, 11(1), 6924.

Cooper, S.M., Scott, H.M., De la Garza, G.R., Deck, A.L. \& Cathey, J.C. (2010). Distribution and interspecies contact of feral swine and cattle on rangeland in south Texas: implications for disease transmission. $J$ Wildlife Dis , 46(1), 152-164.

Couzin, I.D., Krause, J., Franks, N.R. \& Levin, S.A. (2005). Effective leadership and decision-making in animal groups on the move. Nature , 433(7025), 513-516.

Couzin, I.D., Ioannou, C.C., Demirel, G., Gross, T., Torney, C.J., Hartnett, A. et al. (2011). Uninformed Individuals Promote Democratic Consensus in Animal Groups. Science, 334 (6062), 1578-80.

Croft, D.P., Edenbrow, M., Darden, S.K., Ramnarine, I.W., van Oosterhout, C. \& Cable, J. (2011). Effect of Gyrodactylid Ectoparasites on Host Behaviour and Social Network Structure in Guppies Poecilia Reticulata. Behav Ecol Sociobiol, 65(12), 2219-27.

Cross, P.C., Lloyd-Smith, J.O., Johnson, P.L.F. \& Getz, W.M. (2005). Duelling Timescales of Host Movement and Disease Recovery Determine Invasion of Disease in Structured Populations. Ecol Lett, 8(6), 587-95.

Cross, P.C., Creech, T.G., Ebinger, M.R., Manlove, K.R., Irvine, K.,Henningsen, J. et al. (2013). Female Elk Contacts Are Neither Frequency nor Density Dependent. Ecology, 94(9), 2076-86.

Cross, P.C., Prosser, D.J., Ramey, A.M., Hanks, E.M. \& Pepin, K.M. (2019). Confronting Models with Data: The Challenges of Estimating Disease Spillover. Philos T R Soc B, 374 (1782), 20180435.

Cutler, D.R., Edwards Jr, T.C., Beard, K.H., Cutler, A., Hess, K.T., Gibson, J. \& Lawler, J.J. (2007). Random forests for classification in ecology. Ecology , 88(11), 2783-2792.

Davis, S., Begon, M., De Bruyn, L., Ageyev, V.S., Klassovskiy, N.L., Pole, S.B. et al. (2004). Predictive thresholds for plague in Kazakhstan. Science, 304(5671), 736-738.

Diallo, B., Sissoko, D., Loman, N.J., Bah, H.A., Bah, H., Worrell, M.C.,et al. (2016). Resurgence of Ebola virus disease in Guinea linked to a survivor with virus persistence in seminal fluid for more than 500 days. Clin Infect Dis , 63(10), 1353-1356.

Dong, J.G., Ha, S.Y., Jung, J. \& Kim, D. (2021). Emergence of stochastic flocking for the discrete CuckerSmale model with randomly switching topologies. Commun Math Sci , 19 (1), 205-228.

Donnelly, C.A., Woodroffe, R., Cox, D.R., Bourne, J., Gettinby, G., Le Fevre, A.M. et al . (2003). Impact of Localized Badger Culling on Tuberculosis Incidence in British Cattle. Nature, 426(6968), 834-37.

Dorak, S.J., Green, M.L., Wander, M.M., Ruiz, M.O., Buhnerkempe, M.G., Tian, T. et al. (2017). Clay Content and pH: Soil Characteristic Associations with the Persistent Presence of Chronic Wasting Disease in Northern Illinois. Sci Rep-UK, 7(1), 18062.

Dougherty, E.R., Seidel, D.P., Carlson, C.J., Spiegel,O. \& Getz, W. M. (2018). Going through the motions: incorporating movement analyses into disease research. Ecol Lett , 21(4), 588-604.

Drewe, J.A. (2010) Who infects whom? Social networks and tuberculosis transmission in wild meerkats. $P$ R Soc B. 277(1681), 633-642.

Edelhoff, H., Signer, J. \& Balkenhol, N. (2016). Path Segmentation for Beginners: An Overview of Current Methods for Detecting Changes in Animal Movement Patterns. Move Ecol, 4(1), 21.

Farine, D.R. (2013). Animal Social Network Inference and Permutations for Ecologists in R using asnipe. Methods Ecol Evol, 4(12), 1187-94.

Farine, D.R.\& Whitehead, H. (2015). Constructing, Conducting and Interpreting Animal Social Network Analysis. J Anim Ecol, 84(5), 1144-63. 
Farine, D.R. (2017). A guide to null models for animal social network analysis. Methods Ecol Evol , 8 (10), 1309-1320.

Fieberg, J., Signer, J., Smith, B. \& Avgar, T. (2021). A 'How to' Guide for Interpreting Parameters in Habitat-Selection Analyses. J Anim Ecol, 90(5), 1027-43.

Firestone, S.M., Hayama, Y., Bradhurst, R., Yamamoto, T., Tsutsui, T. \& Stevenson, M.A. (2019). Reconstructing Foot-and-Mouth Disease Outbreaks: A Methods Comparison of Transmission Network Models. Sci Rep-UK,9(1), 4809.

Fleming, C.H., Calabrese, J.M., Mueller, T., Olson, K.A., Leimgruber, P. \& Fagan, W.F. (2014). From Fine-Scale Foraging to Home Ranges: A Semivariance Approach to Identifying Movement Modes across Spatiotemporal Scales. Am Nat, 183(5), E154-67.

Fleming, C.H., Fagan, W.F., Mueller, T., Olson, K.A., Leimgruber, P. \& Calabrese, J.M. (2015). Rigorous Home Range Estimation with Movement Data: A New Autocorrelated Kernel Density Estimator. Ecology,96(5), 1182-88.

Fortin, D., Beyer, H.L., Boyce, M.S., Smith, D.W., Duchesne, T. \& Mao, J.S. (2005). Wolves influence elk movements: behavior shapes a trophic cascade in Yellowstone National Park. Ecology, 86(5), 1320-30.

Fortuna, M.A., Popa-Lisseanu, A.G., Ibanez, C. \& Bascompte, J. (2009). The Roosting Spatial Network of a Bird-Predator Bat. Ecology,90(4), 934-44.

Funk, S., Bansal, S., Bauch, C.T., Eames, K.T.D., Edmunds, W.J., Galvani, A.P. \& Klepac, P. (2015). Nine Challenges in Incorporating the Dynamics of Behaviour in Infectious Diseases Models. Epidemics,10 (March), 21-25.

Garlick, M.J., Powell, J.A., Hooten, M.B. \& McFarlane, L.R. (2011). Homogenization of large-scale movement models in ecology. B Math Biol, 73 (9), 2088-2108.

Garcia Fontes, S., Morato, R.G., Stanzani, S.L. \& Correa, P.L.P. (2021). Jaguar Movement Behavior: Using Trajectories and Association Rule Mining Algorithms to Unveil Behavioral States and Social Interactions. PLoS One, 16(2), e0246233.

Gerbier, G., Baldet, T., Tran, A., Hendrickx, G., Guis, H., Mintiens, K., Elbers, A. R. W. \& Staubach, C. (2008). Modelling Local Dispersal of Bluetongue Virus Serotype 8 Using Random Walk." Prev Vet Med,87(1-2), 119-30.

Gilbertson, M.L.J., White, L.A. \& Craft, M.E. (2021). Trade-Offs with Telemetry-Derived Contact Networks for Infectious Disease Studies in Wildlife." Methods Ecol Evol , 12(1), 76-87.

Grear, D.A., Luong, L.T. \& Hudson, P.J. (2013). Network transmission inference: host behavior and parasite life cycle make social networks meaningful in disease ecology. Ecol Appl, 23 (8), 1906-1914.

Groendyke, C., Welch, D., \& Hunter, D. R. (2012). A network-based analysis of the 1861 Hagelloch measles data. Biometrics, 68(3), 755-765.

Grunewalder, S., Broekhuis, F., Macdonald, D.W., Wilson, A.M., McNutt, J.W., Shawe-Taylor, J. et al. (2012). Movement activity based classification of animal behaviour with an application to data from cheetah (Acinonyx jubatus). PLoS One, 7 (11), e49120.

Gurarie, E. \& Ovaskainen, O. (2013). Towards a General Formalization of Encounter Rates in Ecology. Theor Ecol, 6(2), 189-202.

Hallatschek, O. \& Fisher, D.S. (2014). Acceleration of Evolutionary Spread by Long-Range Dispersal. Proc Natl Acad Sci USA, 111(46), E4911-19.

Hall, R.J., Altizer, S. \& Bartel, R.A. (2014). Greater migratory propensity in hosts lowers pathogen transmission and impacts. J Anim Ecol , 83(5), 1068-1077. 
Hall, M.D., Woolhouse, M.E.J. \& Rambaut, A. (2016). Using Genomics Data to Reconstruct Transmission Trees during Disease Outbreaks. Rev Sci Tech OIE, 35(1), 287-96.

Han, B. A., O'Regan, S. M., Paul Schmidt, J. \& Drake, J. M. (2020). Integrating data mining and transmission theory in the ecology of infectious diseases. Ecol Lett , 23(8), 1178-1188.

Hanks, E.M. \& Hooten, M.B. (2013). Circuit Theory and Model-Based Inference for Landscape Connectivity. J Am Stat Assoc, 108(501), 22-33.

Hanks, E.M., Hooten, M.B., Johnson, D.S. \& Sterling, J.T. (2011). Velocity-Based Movement Modeling for Individual and Population Level Inference. PLoS One, 6(8), e22795.

Harari, G.M., Muller, S.R., Aung, M.S. \& Rentfrow, P.J. (2017a). Smartphone sensing methods for studying behavior in everyday life. Current opinion in behavioral sciences , 18, 83-90.

Harari, G.M., Gosling, S.D., Wang, R., Chen, F., Chen, Z. \& Campbell, A.T. (2017b). Patterns of behavior change in students over an academic term: A preliminary study of activity and sociability behaviors using smartphone sensing methods. Comput Hum Behav , 67, 129-138.

Haw, D.J., Pung, R., Read, J.M. \& Riley, S. (2020). Strong Spatial Embedding of Social Networks Generates Nonstandard Epidemic Dynamics Independent of Degree Distribution and Clustering. Proc Natl Acad Sci USA, 117(38), 23636-23642.

Hefley, T.J., Hooten, M.B., Russell, R.E., Walsh, D.P. \& Powell, J.A. (2017). When mechanism matters: Bayesian forecasting using models of ecological diffusion. Ecol Lett , 20(5), 640-650.

Hess, G. (1996). Disease in Metapopulation Models: Implications for Conservation. Ecology, 77(5), 1617-32.

Hetem, R.S., Mitchell, D., Maloney, S.K., Meyer, L.C.R., Fick, L.G., Kerley, G.I.H. \& Fuller, A. (2008). Fever and Sickness Behavior during an Opportunistic Infection in a Free-Living Antelope, the Greater Kudu (Tragelaphus Strepsiceros). Am J Physiol-Reg I, 294(1), R246-54.

Hooten, M.B., Scharf, H.R., Hefley, T.J., Pearse, A.T. \& Weegman, M.D. (2018). Animal Movement Models for Migratory Individuals and Groups. Methods Ecol Evol, 9(7), 1692-1705.

Hughes, D.P., Andersen, S.B., Hywel-Jones, N.L., Himaman, W., Billen, J. \& Boomsma, J.J. (2011). Behavioral mechanisms and morphological symptoms of zombie ants dying from fungal infection. BMC Ecol, 11(1), 1-10.

Jesmer, B.R., Merkle, J.A., Goheen, J.R., Aikens, E.O., Beck, J.L., Courtemanch, A.B., Hurley, M.A. et al - (2018). Is Ungulate Migration Culturally Transmitted? Evidence of Social Learning from Translocated Animals. Science, 361(6406), 1023-25.

Jonsen, I.D., Flemming, J.M. \& Myers, R.A. (2005). Robust State-space Modeling of Animal Movement Data. Ecology, 86(11), 2874-80.

Kamath, P.L., Foster, J.T., Drees, K.P., Luikart, G., Quance, C., Anderson, N.J., Clarke, P.R. et al . (2016). Genomics Reveals Historic and Contemporary Transmission Dynamics of a Bacterial Disease among Wildlife and Livestock. Nat Commun, 7 (May), 11448.

Kays, R., Crofoot, M.C., Jetz, W. \& Wikelski, M. (2015). Terrestrial animal tracking as an eye on life and planet. Science, 348(6240).

Keeling, M.J., Woolhouse, M.E., Shaw, D.J., Matthews, L., Chase-Topping, M., Haydon, D.T. et al. (2001). Dynamics of the 2001 UK Foot and Mouth Epidemic: Stochastic Dispersal in a Heterogeneous Landscape.Science, 294(5543), 813-17.

Keeling, M.J., \& Rohani, P. (2002). Estimating Spatial Coupling in Epidemiological Systems: A Mechanistic Approach. Ecol Lett, 5(1), 20-29. 
Koella, J.C., Sorensen, F.L. \& Anderson, R.A. (1998). The Malaria Parasite, Plasmodium Falciparum, Increases the Frequency of Multiple Feeding of Its Mosquito Vector, Anopheles Gambiae. P R Soc B,265(1398), 763-68.

Kolmogorov, A.N. (1937). A study of the equation of diffusion with increase in the quantity of matter, and its application to a biological problem. Mosc Univ Bull Math , 1, 1-25.

Kraemer, M.U.G., Reiner Jr, R.C., Brady, O.J., Messina, J.P., Gilbert, M., Pigott, D.M., Yi, D. et al - (2019). Past and Future Spread of the Arbovirus Vectors Aedes Aegypti and Aedes Albopictus. Nat Microbiol, 4(5), 854-63.

Kranstauber, B., Kays, R., Lapoint, S.D., Wikelski, M. \& Safi, K. (2012). A Dynamic Brownian Bridge Movement Model to Estimate Utilization Distributions for Heterogeneous Animal Movement. J Anim Ecol,81(4), $738-46$.

Krause, J., Krause, S., Arlinghaus, R., Psorakis, I., Roberts, S. \& Rutz, C. (2013). Reality mining of animal social systems. Trends Ecol Evol, 28(9), 541-551.

Laver, P.N. \& Kelly, M.J. (2008). A Critical Review of Home Range Studies. J Wildlife Manage, 72 (1), 290-98.

Levis, D., Diaz-Guilera, A., Pagonabarraga, I. \& Starnini, M. (2020). Flocking-enhanced social contagion. Phys Rev Res , 2(3), 032056.

Long, J.A., Nelson, T.A., Webb, S.L. \& Gee, K.L. (2014). A Critical Examination of Indices of Dynamic Interaction for Wildlife Telemetry Studies. J Anim Ecol, 83(5), 1216-33.

Lloyd-Smith, J.O., Schreiber, S.J., Kopp, P.E. \& Getz, W.M. (2005). Superspreading and the effect of individual variation on disease emergence. Nature , 438(7066), 355-359.

Manlove, K.R., Cassirer, E.F., Cross, P.C., Plowright, R.K. \& Hudson, P.J. (2014). Costs and benefits of group living with disease: a case study of pneumonia in bighorn lambs (Ovis canadensis). $P R$ Soc B , 281 (1797), 20142331.

Manlove, K.R., Cassirer, E.F., Plowright, R.K., Cross, P.C. \& Hudson, P.J. (2017). Contact and contagion: Probability of transmission given contact varies with demographic state in bighorn sheep. J Anim Ecol , 86(4), 908-920.

Manlove, K.R., Aiello, C., Sah, P., Cummins, B., Hudson, P.J. \& Cross, P.C. (2018). The Ecology of Movement and Behaviour: A Saturated Tripartite Network for Describing Animal Contacts. P $R$ Soc $B, 285(1887), 20180670$

Manlove, K.R., Sampson, L.M., Borremans, B., Cassirer, E.F., Miller, R.S., Pepin, K.M. et al . (2019). Epidemic growth rates and host movement patterns shape management performance for pathogen spillover at the wildlife-livestock interface. Philos T R Soc B , 374(1782), 20180343.

Marien, J., Borremans, B., Verhaeren, C., Kirkpatrick, L., Gryseels, S., Gouy de Bellocq, J. et al. (2020). Density dependence and persistence of Morogoro arenavirus transmission in a fluctuating population of its reservoir host. J Anim Ecol , 89 (2), 506-518.

Marrotte, R.R. \& Bowman, J. (2017). The Relationship between Least-Cost and Resistance Distance. PLoS One, 12(3), e0174212

Martin-Lof, A. (1998). The final size of a nearly critical epidemic, and the first passage time of a Wiener process to a parabolic barrier. J Appl Probab , 35 (3), 671-682.

Martinez-Garcia, R., Fleming, C.H., Seppelt, R., Fagan, W.F. \& Calabrese, J.M. (2020). How Range Residency and Long-Range Perception Change Encounter Rates. J Theor Biol, 498(August), 110267. 
Martiskainen, P., Jarvinen, M., Skon, J.P., Tiirikainen, J., Kolehmainen, M. \& Mononen, J. (2009). Cow behaviour pattern recognition using a three-dimensional accelerometer and support vector machines. Appl Anim Behav Sci , 119(1-2), 32-38.

Masucci, A.P., Serras, J., Johansson, A. \& Batty, M. (2013). Gravity versus Radiation Models: On the Importance of Scale and Heterogeneity in Commuting Flows. Phys Rev E, 88(2), 022812.

Matthiopoulos, J., Hebblewhite, M., Aarts, G. \& Fieberg, J. (2011). Generalized functional responses for species distributions.Ecology, 92, 583-589.

McClintock, B.T., King, R., Thomas, L., Matthiopoulos, J., McConnell, B.J. \& Morales, J.M. (2012). A General Discrete-Time Modeling Framework for Animal Movement Using Multistate Random Walks. Ecol Monogr,82(3), 335-349.

McClintock, B.T., Johnson, D.S., Hooten, M.B., Ver Hoef, J.M. \& Morales, J.M. (2014). When to be discrete: the importance of time formulation in understanding animal movement. Move Ecol , 2(1), 1-14.

McComb, K., Moss, C., Sayialel, S. \& Baker, L. (2000). Unusually extensive networks of vocal recognition in African elephants. Anim Behav , 59(6), 1103-1109.

Meekan, M.G., Duarte, C.M., Fernandez-Gracia, J., Thums, M., Sequeira, A.M., Harcourt, R. et al. (2017). The ecology of human mobility. Trends Ecol Evol , 32(3), 198-210

Merkle, J.A., Cross, P.C., Scurlock, B.M., Cole, E.K., Courtemanch, A.B., Dewey, S.R. et al . (2018). Linking Spring Phenology with Mechanistic Models of Host Movement to Predict Disease Transmission Risk. Edited by Jason Rohr. J Appl Ecol, 55(2), 810-19.

Meyer, S., Elias, J., \& Hohle, M. (2012) A space-time conditional intensity model for invasive meningococcal disease occurrence. Biometrics , 68(2), 607-616.

Miller, H.J., Dodge, S., Miller, J. \& Bohrer, G. (2019). Towards an integrated science of movement: converging research on animal movement ecology and human mobility science. Int J Geogr Inf Sci, 33(5), $855-876$.

Moorcroft, P.R. \& Barnett, A. (2008). Mechanistic Home Range Models and Resource Selection Analysis: A Reconciliation and Unification.Ecology, 89(4), 1112-19.

Morales, J.M., Haydon, D.T., Frair, J., Holsinger, K.E. \& Fryxell, J.M. (2004). Extracting More out of Relocation Data: Building Movement Models as Mixtures of Random Walks. Ecology, 85(9), 2436-45.

Moreau, G., Fortin, D., Couturier, S. \& Duchesne, T. (2012). Multi-level functional responses for wildlife conservation: the case of threatened caribou in managed boreal forests. J Appl Ecol . 49, 611-620.

Morton, A. \& Finkenstadt, B.F. (2005). Discrete Time Modelling of Disease Incidence Time Series by Using Markov Chain Monte Carlo Methods.J R Stat Soc C-Appl , 54(3), 575-94.

Muff, S., Signer, J. \& Fieberg, J. (2020). Accounting for Individual-Specific Variation in Habitat-Selection Studies: Efficient Estimation of Mixed-Effects Models Using Bayesian or Frequentist Computation. J Anim Ecol, 89(1), 80-92.

Mysterud, A. \& Ims, R.A. (1998). Functional Responses in Habitat Use: Availability Influences Relative Use in Trade-off Situations.Ecology, 79(4), 1435-41.

Mysterud, A., Skjelbostad, I.N., Rivrud, I.M., Brekkum, O. \& Meisingset, E.L. (2021). Spatial Clustering by Red Deer and Its Relevance for Management of Chronic Wasting Disease. Animals , 11(5), 1272.

Nathan, R., Getz, W.M., Revilla, E., Holyoak, M., Kadmon, R., Saltz, D.et al (2008). A movement ecology paradigm for unifying organismal movement research. Proc Natl Acad Sci USA, 105(49), 19052-19059. 
Nathan, R., Spiegel, O., Fortmann-Roe, S., Harel, R., Wikelski, M. \& Getz, W. M. (2012). Using tri-axial acceleration data to identify behavioral modes of free-ranging animals: general concepts and tools illustrated for griffon vultures. J Exp Biol, 215(6), 986-996.

Noonan, M.J., Tucker, M.A., Fleming, C.H., Akre, T.S., Alberts, S.C., Ali, A.H. Altmann, J. et al. (2019). A Comprehensive Analysis of Autocorrelation and Bias in Home Range Estimation." Ecol Monogr,89(2), $\mathrm{e} 01344$.

Northrup, J.M., Avrin, A., Anderson, C.R., Brown, E. \& Wittemyer, G. (2019). On-Animal Acoustic Monitoring Provides Insight to Ungulate Foraging Behavior. J Mammal, 100(5), 1479-89.

O'Brien, J.M., O'Brien, C.S., McCarthy, C. \& Carpenter, T.E. (2014). Incorporating Foray Behavior into Models Estimating Contact Risk between Bighorn Sheep and Areas Occupied by Domestic Sheep. Wildlife Soc B, 38(2), 321-31.

Oraby, T., Vasilyeva, O., Krewski, D. \& Lutscher, F. (2014). Modeling Seasonal Behavior Changes and Disease Transmission with Application to Chronic Wasting Disease. J Theor Biol, 340, 50-59.

Palla, G., Derenyi, I., Farkas, I. \& Vicsek, T. (2005). Uncovering the overlapping community structure of complex networks in nature and society. Nature, 435(7043), 814-818.

Park, A.W., Gubbins, S. \& Gilligan, C.A. (2002). Extinction times for closed epidemics: the effects of host spatial structure. Ecol Lett , 5(6), 747-755.

Pedersen, M.W., Patterson, T.A., Thygesen, U.H. \&Madsen, H. (2011). Estimating animal behavior and residency from movement data. Oikos , 120(9), 1281-1290.

Peel, A.J., Pulliam, J.R.C., Luis, A.D., Plowright, R.K., O'Shea, T.J., Hayman, D.T.S. et al. (2014). The Effect of Seasonal Birth Pulses on Pathogen Persistence in Wild Mammal Populations. P R Soc B,281(1786).

Pepin, K.M., Golnar, A. \& Podgorski, T. (2021). Social Structure Defines Spatial Transmission of African Swine Fever in Wild Boar.J R Soc Interface, 18(174), 20200761.

Pepin, K.M., Kay, S.L., Golas, B.D., Shriner, S.S., Gilbert, A.T., Miller, R.S. et al. (2017). Inferring Infection Hazard in Wildlife Populations by Linking Data across Individual and Population Scales. Ecol Lett, 20(3), 275-92.

Pinter-Wollman, N., Fiore, S.M. \& Theraulaz, G. (2017). The impact of architecture on collective behaviour. Nat Ecol Evol , 1(5), 1-2.

Plowright, R.K., Foley, P., Field, H.E., Dobson, A.P., Foley, J.E., Eby, P. et al. , (2011). Urban habituation, ecological connectivity and epidemic dampening: the emergence of Hendra virus from flying foxes (Pteropus spp.). P R Soc B , 278(1725), 3703-3712.

Plowright, R.K., Becker, D.J., McCallum, H. \& Manlove, K.R. (2019). Sampling to elucidate the dynamics of infections in reservoir hosts. Philos T R Soc B , 374(1782), 20180336.

Podgorski, T., Baś, G., Jędrzejewska, B., Sönnichsen, L., Śnieżko, S., Jędrzejewski, W. et al. (2013). Spatiotemporal Behavioral Plasticity of Wild Boar (Sus Scrofa) under Contrasting Conditions of Human Pressure: Primeval Forest and Metropolitan Area. J Mammal , 94(1), 109-119.

Potts, J.R., Bastille-Rousseau, G., Murray, D.L., Schaefer, J.A. \& Lewis, M.A. (2014). Predicting Local and Non-Local Effects of Resources on Animal Space Use Using a Mechanistic Step Selection Model.Methods Ecol Evol, 5(3), 253-62.

Prager, K.C., Buhnerkempe, M.G., Greig, D.J., Orr, A.J., Jensen, E.D., Gomez, F. et al . (2020). Linking Longitudinal and Cross-Sectional Biomarker Data to Understand Host-Pathogen Dynamics: Leptospira in California Sea Lions (Zalophus Californianus) as a Case Study.PLoS Neglect Trop D, 14(6), e0008407. 
Prosser, D.J., Palm, E.C., Takekawa, J.Y., Zhao, D., Xiao, X., Li, P.et al . (2016). Movement Analysis of Free-Grazing Domestic Ducks in Poyang Lake, China: A Disease Connection. Int J Geogr Inf Sci, 30(5), 869-80.

Remais, J., Akullian, A., Ding, L. \& Seto, E. (2010). Analytical methods for quantifying environmental connectivity for the control and surveillance of infectious disease spread. $J$ R Soc Interface , 7 (49), 11811193.

Reynolds, D.S., Shoemaker, K., von Oettingen, S. \& Najjar, S. (2017). High rates of winter activity and arousals in two New England bat species: implications for a reduced White-nose Syndrome impact? Northeast Nat , $24(\operatorname{sp} 7)$.

Richardson, T.O. \& Gorochowski, T.E. (2015). Beyond Contact-Based Transmission Networks: The Role of Spatial Coincidence. J R Soc Interface, 12(111), 20150705.

Riley, S. \& Ferguson, N.M. (2006). Smallpox Transmission and Control: Spatial Dynamics in Great Britain. Proc Natl Acad Sci USA,103(33), 12637-42.

Roberts, M. G. (2007). The Pluses and Minuses of R0. J R Soc Interface, 4(16), 949-61.

Rohani, P., Breban, R., Stallknecht, D.E. \& Drake, J.M. (2009). Environmental transmission of low pathogenicity avian influenza viruses and its implications for pathogen invasion. Proc Natl Acad Sci USA , 106(25), 10365-10369.

Rosatte, R., Sobey, K., Donovan, D., Bruce, L., Allan, M., Silver, A.et al. (2006). Behavior, Movements, and Demographics of Rabid Raccoons in Ontario, Canada: Management Implications. J Wildlife Dis, 42(3), 589-605.

Sah, P., Leu, S.T., Cross, P.C., Hudson, P.J. \& Bansal, S. (2017). Unraveling the Disease Consequences and Mechanisms of Modular Structure in Animal Social Networks. Proc Natl Acad Sci USA, 114(16), 4165-70.

Salkeld, D.J., Salathé, M., Stapp, P. \& Jones, J.H. (2010). Plague outbreaks in prairie dog populations explained by percolation thresholds of alternate host abundance. Proc Natl Acad Sci USA , 107(32), 1424714250 .

Scherer, C., Radchuk, V., Franz, M., Thulke, H-H., Lange, M., Grimm, V.et al. (2020). Moving Infections, Individual Movement Decisions Drive Disease Persistence in Spatially Structured Landscapes. Oikos, 129(5), 651-67.

Shaw, A.K., Sherman, J., Barker, F.K. \& Zuk, M. (2018). Metrics Matter: The Effect of Parasite Richness, Intensity and Prevalence on the Evolution of Host Migration. $P R$ Soc B, 285(1891).https://doi.org/10.1098/rspb.2018.2147 .

Shoemaker, K.T., Heffelfinger, L.J., Jackson, N.J., Blum, M.E., Wasley, T. \& Stewart, K.M. (2018). A machine-learning approach for extending classical wildlife resource selection analyses. Ecol Evol , 8(6), 35563569 .

Sih, A., Spiegel, O., Godfrey, S., Leu, S. \& Bull, C. M. (2018). Integrating social networks, animal personalities, movement ecology and parasites: a framework with examples from a lizard. Animal Behav , 136, 195-205.

Silk, M.J., Finn, K.R., Porter, M.A. \& Pinter-Wollman, N. (2018). Can multilayer networks advance animal behavior research? Trends Ecol Evol , 33(6), 376-378.

Simini, F., Gonzalez, M.C., Maritan, A. \& Barabasi, A-L. (2012). A Universal Model for Mobility and Migration Patterns. Nature,484(7392), 96-100.

Skellam, J.G. (1951). Random dispersal in theoretical populations. Biometrika, 38(1/2), 196-218. 
Sokolow, S.H., Nova, N., Pepin, K.M., Peel, A.J., Pulliam, J.R.C., Manlove, K. et al. (2019). Ecological Interventions to Prevent and Manage Zoonotic Pathogen Spillover. Philos T R Soc B,374(1782), 20180342.

Strandburg-Peshkin, A., Twomey, C.R., Bode, N.W.F., Kao, A.B., Katz, Y., Ioannou, C.C. et al. (2013). Visual sensory networks and effective information transfer in animal groups. Curr Biol, 23(17), R709-R711.

Stockmaier, S., Stroeymeyt, N., Shattuck, E.C., Hawley, D.M., Meyers, L.A. \& Bolnick, D.I. (2021). Infectious Diseases and Social Distancing in Nature. Science, 371(6533).

Tarlo, S.M., Altman, K.W., Oppenheimer, J., Lim, K., Vertigan, A., Prezant, D. et al. (2016). Occupational and environmental contributions to chronic cough in adults: chest expert panel report. Chest , 150(4), 894907.

Teitelbaum, C.S., Huang, S., Hall, R.J. \& Altizer, S. (2018). Migratory behaviour predicts greater parasite diversity in ungulates. $P R$ Soc $B, 285(1875), 20180089$.

Tizzoni, M., Bajardi, P., Decuyper, A., King, G.K.K., Schneider, C.M., Blondel, V. et al. (2014). On the Use of Human Mobility Proxies for Modeling Epidemics. PLoS Comput Biol, 10(7), e1003716.

Townsend, A.K., Hawley, D.M., Stephenson, J.F. \& Williams, K.E.G. (2020). Emerging Infectious Disease and the Challenges of Social Distancing in Human and Non-Human Animals. $P R$ Soc B, 287(1932), 20201039 .

Tsao, K., Sellman, S., Beck-Johnson, L.M., Murrieta, D.J., Hallman, C., Lindstrom, T. et al . (2020). Effects of Regional Differences and Demography in Modelling Foot-and-Mouth Disease in Cattle at the National Scale. Interface Focus, 10(1), 20190054.

Turner, W.C., Kausrud, K.L., Krishnappa, Y.S., Cromsigt, J.P.G.M., Ganz, H.H., Mapaure, I. et al. (2014). Fatal Attraction: Vegetation Responses to Nutrient Inputs Attract Herbivores to Infectious Anthrax Carcass Sites. P R Soc B, 281(1795), 20141785.

van Beest, F.M., Uzal, A., Vander Wal, E., Laforge, M.P., Contasti, A.L., Colville, D. \& McLoughlin, P.D. (2014). Increasing density leads to generalization in both coarse-grained habitat selection and fine-grained resource selection in a large mammal. J Anim Ecol , 83(1), 147-156.

Van Ness, G.B. (1971). Ecology of anthrax. Science, 172(3990), 1303-1307

Viboud, C., Bjornstad, O.N., Smith, D.L., Simonsen, L., Miller, M.A. \& Grenfell, B.T. (2006). Synchrony, Waves, and Spatial Hierarchies in the Spread of Influenza. Science, 312(5772), 447-451.

Vyas, A., Kim, S-K., Giacomini, N., Boothroyd, J.C. \& Sapolsky, R.M. (2007). Behavioral Changes Induced by Toxoplasma Infection of Rodents Are Highly Specific to Aversion of Cat Odors. Proc Natl Acad Sci USA, 104(15), 6442-47.

Wang, G. (2019). Machine learning for inferring animal behavior from location and movement data. Ecol Inform, 49 , pp.69-76.

Wasserberg, G., Osnas, E.E., Rolley, R.E. \& Samuel, M.D. (2009). Host Culling as an Adaptive Management Tool for Chronic Wasting Disease in White-Tailed Deer: A Modelling Study. J Appl Ecol, 46(2), 457-66.

Webber, Q.M.R. \& Vander Wal, E. (2018). An Evolutionary Framework Outlining the Integration of Individual Social and Spatial Ecology.J Anim Ecol, 87(1), 113-27.

Webber, Q.M., \& Willis, C.K. (2020). Personality affects dynamics of an experimental pathogen in little brown bats. Roy Soc Open Sci , 7(9), 200770.

Weinstein, S.B., Buck, J.C. \& Young, H.S. (2018). A landscape of disgust. Science, 359(6381), 1213-1214.

Weiss, G.H. \& Dishon, M. (1971). On the asymptotic behavior of the stochastic and deterministic models of an epidemic. Math Biosci , 11(3-4), 261-265. 
White, L.A., Forester, J.D. \& Craft, M.E. (2018). Disease Outbreak Thresholds Emerge from Interactions between Movement Behavior, Landscape Structure, and Epidemiology. Proc Natl Acad Sci USA, 115(28), $7374-79$.

Wilber, M., Yang, A., Boughton, R., Manlove, K., Miller, R., Pepin, K. \& Wittemyer, G. (in review ) A framework for leveraging animal movement to understand spatial disease dynamics.

Wijeyakulasuriya, D.A., Eisenhauer, E.W., Shaby, B.A. \& Hanks, E.M. (2020). Machine learning for modeling animal movement. PLoS One, 15(7), e0235750.

Wijeyakulasuriya, D.A., Hanks, E.M., Shaby, B.A. \& Cross, P.C. (2019). Extreme Value-Based Methods for Modeling Elk Yearly Movements. J Arg Biol Envir S, 24(1), 73-91.

Yang, A., Schlichting, P., Wight, B., Anderson, W.M., Chinn, S.M., Wilber, M.Q. et al . (2021). Effects of social structure and management on risk of disease establishment in wild pigs. J Anim Ecol, 90(4), 820-833.

Yang, A., Boughton, R.K., Miller, R.S., Wight, B., Anderson, W.M., Beasley, J.C. et al. (2021b). Spatial variation in direct and indirect contact rates at the wildlife-livestock interface for informing disease management. Prev Vet Med , 194, 105423

Zipf, G.K. (1946). The P 1 P 2/D hypothesis: on the intercity movement of persons. Am Sociol Rev , 11(6), 677-686.

Figure legends

Figure 1. Conceptual integration of environment, movement, and pathogen transmission. (1a) and (1b) Mechanistic drivers link environmental context to host movements and pathogen life histories, producing sets of traits that could be inferred via conventional methods or defined a priori to serve as features for machine learning. (2) Pathogen and host movement traits define an epidemiological landscape, consisting of host density, mobility, and contact patterns. These in turn generate patterns in spatial epidemiology, captured through both spatially explicit dynamic models and spatial transmission traits. When data on spatial transmission traits exist, they can act as a basis for assessing the importance of the density, mobility, and contact processes (red arrows from (3) to (2)). If density, mobility, and contact (2) are directly measured, they can feed forward into models of transmission; otherwise they can be inferred or predicted using the mechanisms and features identified in stages (1a) and (1b).

Figure 2. A strategy to improve integration of movement and disease. Movement insights for disease ecology can be grouped into two priority areas - linking environment to spatial ecology of hosts and pathogens, and linking host social ecology to patterns of mobility and contact (A). Modern inferential tools in movement ecology offer opportunities to relate high-resolution descriptions of an animal's movements to drivers in both priority areas (C). However, in each case, the integration of movement insights with epidemiological models hinges on newly emergent or as-yet-undeveloped methods that connect movement inference to critical aspects of the epidemiological landscape (B). Overcoming the gaps will allow for direct prediction of the epidemiological landscape from base principles of movement, improving the transportability of predictions in spatial disease dynamics.

Figure 3. Hypothetical workflows using the epidemiological landscape. Each workflow uses animal movement trajectories to forecast movements from environmental covariates, adjusts movement forecasts according to time lags imposed by pathogen life-history, and integrates with disease models (Box 1) to predict spatiotemporal transmission dynamics. Auxiliary data can enter the workflows (examples indicated by steps flagged with dark green arrows), but may limit model transportability. Many of the methods already exist (checkmarks), though it is not always clear how they should be connected. Other methods have been proposed and prototyped, but are as-yet untested on real-word data (blue triangles). A third group remains strictly hypothetical (red stars). "RSF" = resource selection function; "SSF" = step selection function; "CTMM" = continuous time movement model; "UD" = utilization distribution; "CTMC" = continuous time 
Markov chain. "MoveSTIR" accounts for temporal lags between pathogen deposition and acquisition (Wilber et al. in review). Method details are in Table 2.

Boxes

\section{Box 1: Spatial models of pathogen transmission}

Partial Differential Equations (PDEs) describe the size of the infected class over continuous space and time. Theirhost density models are continuous, and often homogeneous (but see Garlick et al. 2011, Hefley et al. 2017). Themobility model ${ }^{1}$ is a spatial diffusion rate and a corresponding functional form. Outputs include existence, structure, and speed of traveling epidemic waves and spatially explicit times to epidemic peak. Assumptions:animals move according to the kernel, which is often isotropic and independent of environment; transmission occurs locally.

Semi-spatial and static network models allow pairwise interactions within local neighborhoods. The host densitymodel is implicit, but relies on discrete units with corresponding disease states. The mobility model is defined through pairwise coupling coefficients between the "locations", along with a specified "neighborhood" with which each location interacts. Outputs are usually derived from a master equation or simulation. Assumptions: known network structure and disease status; a priori definition of "neighborhood" (depending on analytical approach).

Metapopulation models track disease dynamics at physical locations coupled with one another across space. The host density and mobility models mirror those of semi-spatial models, though locations are now spatially explicit, and mobility can include explicit functions of geographic distance. Outputsinclude spatial spreading rate, spatial synchrony among subunits, and individual- and patch-level reproductive numbers. Assumptions: a priori knowledge of system connectivity.

$I B M s$ allow movement and transmission to emerge organically from predefined rules applied to a set of actors. Inputs are individual-level attributes and parameters that govern them. Thehost density model can be continuous or discrete. Themobility model typically allows an individual's state and environment to interact through a set of movement rules. Outputs range from a simple wave front of disease spread to each individual's spatiotemporally explicit contribution to reproductive numbers. Assumptions: depend on model specifics.

Spatially embedded social networks describe disease dynamics across multipartite networks whose nodes correspond explicitly to locations in space. Inputs are bipartite networks linking individuals to locations of different types (households, peer groups, etc.), The host density model is a set of spatial centroids associated with each group, and mobility models can be distance-, gravity-, or radiation-based. Outputsinclude estimates of $\mathrm{R}_{0}$, total epidemic size, and spatial and temporal patterns of transmission. Assumptions: constant connectivities; central-place space use patterns.

${ }^{1}$ The contact process is often subsumed into a constant transmission rate or absorbed into themobility model

\section{Hosted file}

EpiLandscape_Table1.docx available at https://authorea.com/users/441732/articles/542163defining-an-epidemiological-landscape-by-connecting-host-movement-to-pathogentransmission

\section{Hosted file}

EpiLandscape_Table2.docx available at https://authorea.com/users/441732/articles/542163defining-an-epidemiological-landscape-by-connecting-host-movement-to-pathogen-

transmission 


\section{1a. Host-environment interface}

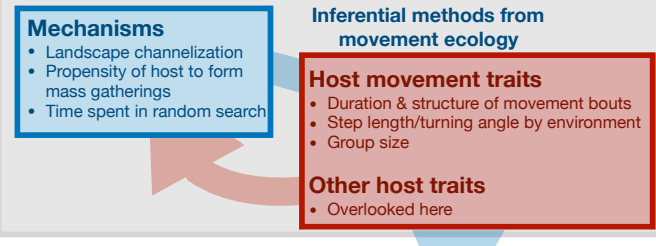

2. Epidemiological landscape

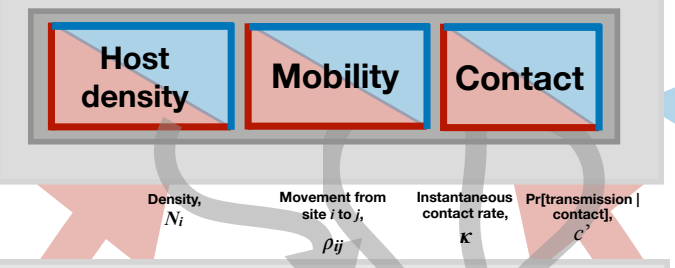

1b. Pathogenenvironment interface

Mechanisms

- Mode of transmission

- Resilience across environments

- Ability to alter host behavior and

movement

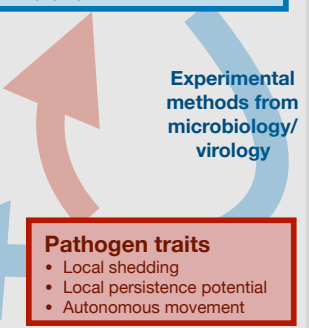

3. Spatial epidemiology

$$
\lambda_{i}=\left(\sum_{j} Y_{j}\left(\frac{1}{N_{i}}\right) \rho_{i j}\right)\left(\kappa c^{\prime}\right)_{i}
$$

Spatially explicit dynamic model

Spatial transmission traits

- Epidemic wave speed and structure

- Overdispersion in number of cases individu

- Location and timing of transmission hotspots
Legend

Phenomenological entity

Mechanistic entity

Mechanistic/

Phenomenological choice

Inferred from mechanism using standard testing

Suggested from pattern

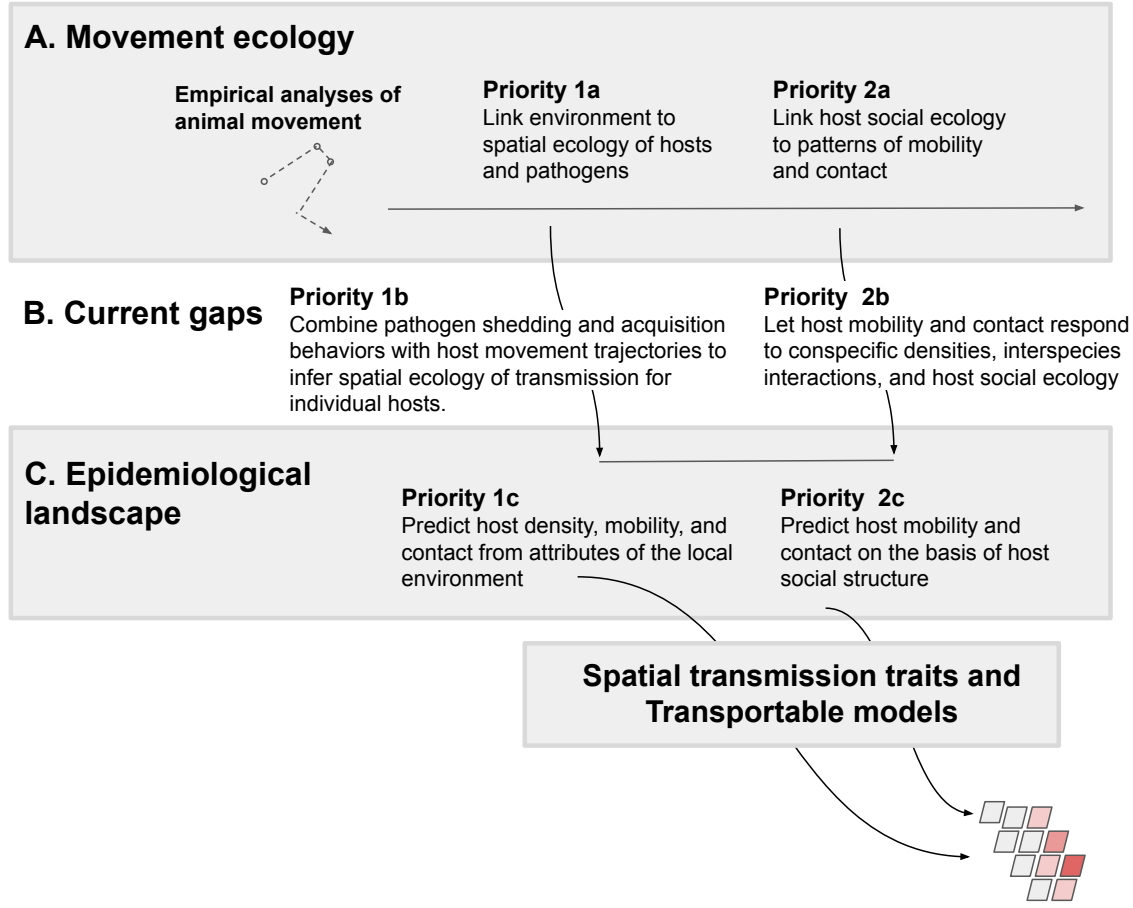




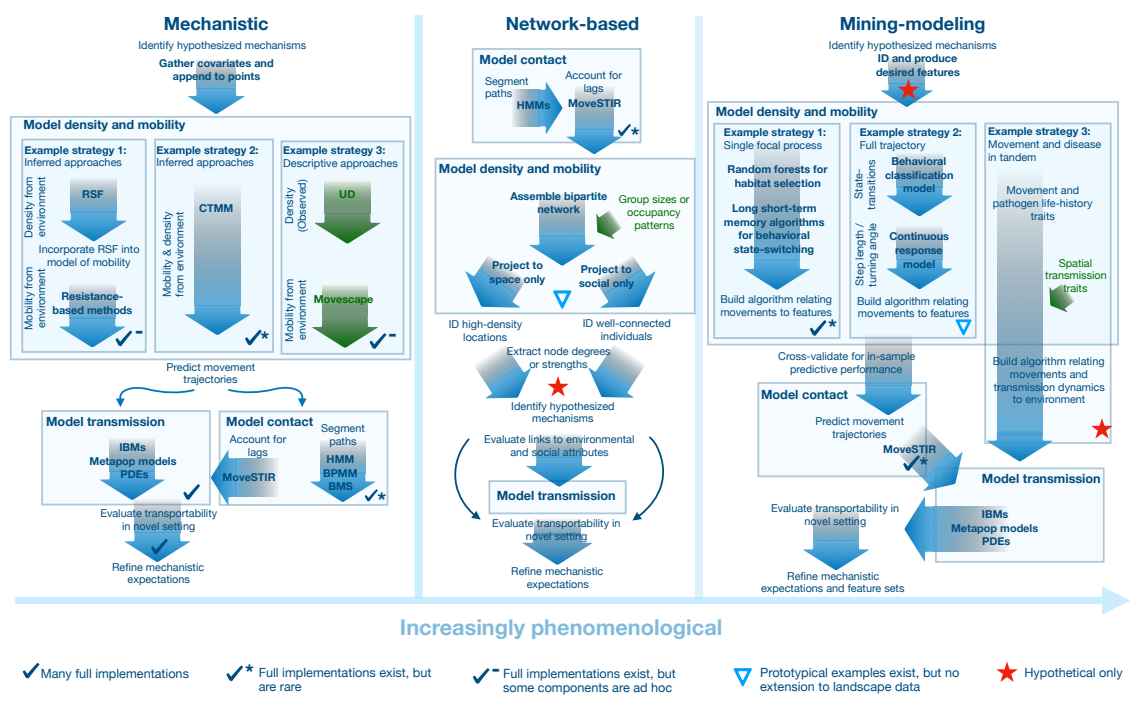

